\title{
GW23-e1508 ANALYSING THE RELATIVE FACTORS THAT INFLUENCE GLOMERULAR FILTRATION RATE IN PRIMARY ALDOSTERONISM.
}

doi:10.1136/heartjnl-2012-302920r.21

Zeng Jian, Wang Xin-ling, Zu Fei-ya, Wang Xin-ling. Hypertension Institute of Xinjiang

Objectives TO analyse the risk factors related to low glomerular filtration rate (GFR) in aldosteronism and to comparing the value of glomerular filtration rate between patients with primary aldosteronism and with essential hypertension.

Methods 189 patients with PA were included in this study. The value of GFR was caculated by MDRD equation, Clinical characteristics, such as the concentration of plasma aldosterone, triglycerides, plasma basic glucose et al were assessed in 198 PA patients and 198 matched controls with EH. The duration of disease, body mass index, blood pressure, concentration of creatnine, GFR and Cysc protein of two groups were studied by two independent samples $\mathrm{T}$ test. Regression analysis were used to find the risk factor of PA that influenced GFR.

\section{Results}

1. both of plasma the concentration of creatnine and Cysc protein in PA are higher than EH group. $(p<0.001)$; eGFR was significant higher in $\mathrm{EH}$ patients than PA patients $(p<0.001)$, and Plasm aldosterone was lower in EH patients $(p<0.05)$.

2. 24-h urine albumin excretion wrer studied by nonparametic test the ruslt shows 24-h urine albumin excretion of PA group is higher than EH group. $(p<0.05)$ By multiple regression analysis, we obtain equation $\mathrm{Y}=420.28-53.83$ Xcystatin C-21.9X 24-h urine albumin- $0.55 \mathrm{X}$ the concentration of plasma aldosterone1.77X the concentration of plasma sodium. The equation suggest that cystatin C, 24-h urine albumin excretion, the concentration of plasma aldosterone and the concentration of plasma sodium are independently predictors of GFR $(p<0.05)$.

Conclusions the results suggest that cystatin C, 2 4-h urine albumin excretion, the concentration of plasma aldosterone are 


\section{ABSTRACTS}

risk factor of GFR. The renal damage is more serious in PA patients. 